\title{
GCS 15: when mild TBI isn't so mild
}

\author{
Latha Ganti ${ }^{1,2^{*}}$, Tej Stead ${ }^{3}$, Yasamin Daneshvar ${ }^{4}$, Aakash N. Bodhit ${ }^{5}$, Christa Pulvino ${ }^{6}$, Sarah W. Ayala ${ }^{7}$ and
}

Keith R. Peters ${ }^{8}$

\begin{abstract}
Objective: The present study characterizes patients with the mildest of mild traumatic brain injury (TBI), as defined by a Glasgow coma score (GCS) of 15.

Methods: This is an IRB approved observational cohort study of adult patients who presented to the emergency department of a Level-1 trauma center, with the primary diagnosis of TBI and a GCS score of 15 on arrival. Data collected included demographic variables such as age, gender, race, mechanisms of injury, signs and symptoms including associated vomiting, seizures, loss of consciousness (LOC), alteration of consciousness (AOC), and posttraumatic amnesia (PTA).Pre- hospital GCS, Emergency Department (ED) GCS, and results of brain CT scans were also collected as well as patient centered outcomes including hospital or intensive care unit (ICU) admission, neurosurgical intervention, and in hospital death. Data were stored in REDCap (Research Electronic Data Capture), a secure, web- based application. Descriptive and inferential analysis was done using JMP 14.0 for the Mac.
\end{abstract}

Results: Univariate predictors of hospital admission included LOC, AOC, and PTA, all $p<0.0001$. Patients admitted to ICU were significantly more likely to be on an antiplatelet or anticoagulant $(P<0.0001)$, have experienced PTA $(p=0.0025)$, LOC $(p<0.0001)$, or have an abnormal brain CT $(p<0.0001)$. Patients who died in the hospital were significantly more likely to be on an antiplatelet or anticoagulant $P=0.0005$. All who died in the hospital had intracranial hemorrhage on ED head CT, despite having presented to the ED with GCS of 15. Patients were also significantly more likely to have had vomiting $(p<0.0001)$. Patients who underwent neurosurgical intervention were significantly more likely to be male $(P=0.0203)$, to be on an antiplatelet or anticoagulant $(P=<0.0001)$ likely to have suffered their TBI from a fall $(P=0.0349)$, and experienced vomiting afterwards $(P=0.0193)$.

Conclusions: This study underscores: 1 ) the importance of neuroimaging in all patients with TBI, including those with a GCS 15. Fully $10 \%$ of our cohort was not imaged. Extrapolating, these would represent $2.5 \%$ bleeds, and $1.47 \%$ fractures. 2) The limitations of GCS in classifying TBI, as patients with even the mildest of mild TBI have a high frequency of gross CT abnormalities.

\section{Introduction}

Traumatic brain injury (TBI) accounts for over 1 million US emergency department visits annually [1], 275,000 civilian hospitalizations [2] and 21,000 military [3] injuries. Traumatic brain injury can have lasting consequences with neurocognitive deficits [4-7], post concussive symptoms [8-10], and repeat return visits to the emergency department [11].

Traumatic brain injury has traditionally been classified as mild, moderate and severe based on the

\footnotetext{
*Correspondence: lathagantimd@gmail.com; latha.ganti@ucf.edu ${ }^{1}$ UCF HCA Emergency Medicine Residency Program of Greater Orlando, University of Central Florida College of Medicine, Orlando, FL, USA ${ }^{2}$ Polk County Fire Rescue, University of Central Florida, Orlando, FL, USA Full list of author information is available at the end of the article
}

Glasgow Coma Scale (GCS), a scoring system never intended to classify brain injury per se but rather level of consciousness. Developed originally in 1974 [12] then modified in 1976 [13], the GCS consists of eye opening, motor, and verbal components, for a total of 15 points. A TBI consensus workgroup points out that while the GCS can be useful in the clinical management and prognosis of TBI, it "does not provide specific information about the pathophysiologic mechanisms which are responsible for the neurologic deficits and targeted by interventions [14]."

Nonetheless, this score is still widely used today. While a GCS of 13 to 15 is considered mild traumatic brain injury (mTBI) per the American College of Rehabilitation Medicine [15], studies have shown that an

(C) The Author(s). 2019 Open Access This article is distributed under the terms of the Creative Commons Attribution 4.0 International License (http://creativecommons.org/licenses/by/4.0/), which permits unrestricted use, distribution, and 
Table 1 cohort demographics

\begin{tabular}{ll}
\hline age & median = 37 years; IQR =23-57; range 18-101. \\
gender & $57 \%$ male \\
race & $83 \%$ white; 14\% black; 3\% hispanic \\
marital status & $61 \%$ single, 27\% married, 7\% divorced or separated, 6\% unknown \\
taking an anti-thrombotic & $\begin{array}{l}11 \% \text { on some agent. 3\% warfarin; 7\% aspirin; 3\% clopidogrel; < 1\% each of heparin, low molecular weight heparin, } \\
\text { agent? }\end{array}$ \\
mechanism of injury & fall 48\%, motor vehicle collision 34\%, assault 30\% \\
came via ambulance? & $62 \%$ \\
\hline
\end{tabular}

mTBI with GCS 13 is not the same as one with GCS 15 [16]. Indeed, even in patients who have a GCS of 15, the mTBI is not always benign. This study characterizes those patients with the mildest of $\mathrm{mTBI}$, as defined by a GCS of 15, and describes the acute injury features, as well as clinical outcomes.

\section{Methods}

This study is derived from a subset of data from a previously published cohort study [17] which included adult patients who presented to the emergency department (ED) of a Level-1 trauma center, over an 18-month period with the primary complaint of TBI (ascertained using ICD-9 codes of 800-804.9, 850-854, and 959.01). For the current study, the patients had to have a GCS of 15 upon ED arrival, and the injury had to have occurred within $24 \mathrm{~h}$ prior to presentation. Demographic variables such as age, gender, past medical history, and medications were abstracted, in addition to the mechanism of injury, and associated signs and symptoms such as vomiting, seizures, loss of consciousness (LOC), alteration of consciousness (AOC), and post- traumatic amnesia (PTA). The patient was considered to have AOC if the neurologic exam revealed a decreased mental status, or if the patient reported feeling dazed or confused or having difficulty thinking. Varibales collected are summarized in the Table 6 in Appendix.
The prehospital and emergency department (ED) GCS were also recorded, as well as vital signs and computed tomography $(\mathrm{CT})$ scan results. This study was approved by our Institutional Review Board. Data were stored in Research Electronic Data Capture (REDCap), a secure web-based application. Descriptive and inferential data analysis was done using JMP 14.0 for Mac.

\section{Results}

The cohort $(n=2211)$ was $57 \%$ male. The marital status was $61 \%$ single, $27 \%$ married, $7 \%$ divorced or separated, and $6 \%$ unknown. The median age was 37 years $(\mathrm{IQR}=$ 23-57), with a range of 18-101 years. Cohort demographics are summarized in Table 1.

The most common symptomatology associated with a GCS of 15 was LOC followed by PTA and AOC. The most common mechanism of injury was fall at $48 \%$, followed by motor vehicle collision at 34\%, and assault or being struck in the head at $30 \%$. The most common location of injury reported was on the road (39\%), followed by inside a home (21\%). The frequency of symptoms is summarized in Fig. 1, and clinical outcomes are summarized in Fig. 2.

More than half the cohort (55\%) reported no alcohol in the $12 \mathrm{~h}$ preceding the head injury, while $17 \%$ admitted to drinking, and another $27 \%$ were "unknown". Alcohol levels were obtained in only 233 patients, or $10 \%$ of

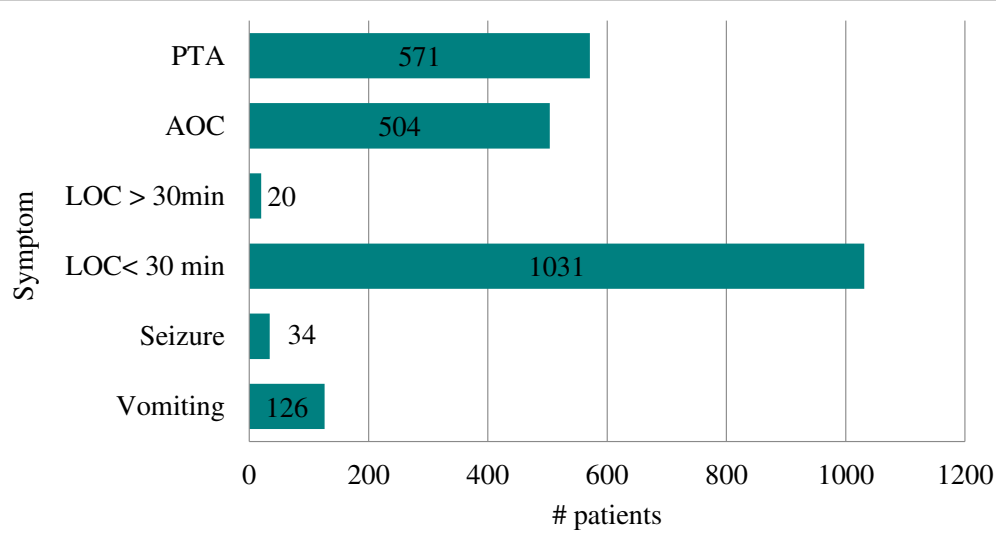

Fig. 1 Frequency of symptoms 


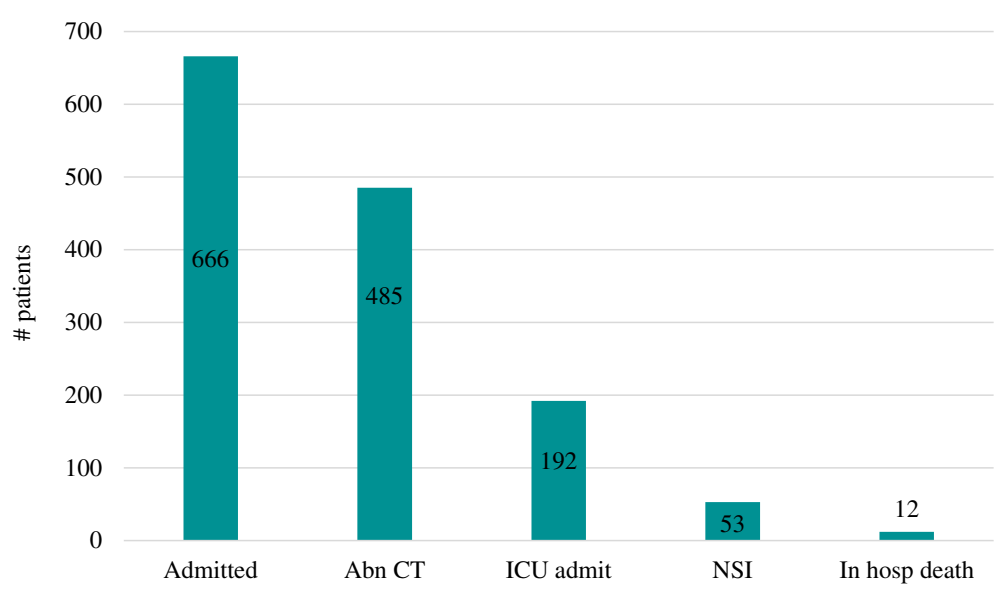

Fig. 2 Frequency of Clinical Outcomes

the cohort. The range was from 0 to 441 , with a median of 153, and an IQR of 37-241. Interestingly, a higher alcohol level was significantly associated with not being admitted to the hospital $(P<0.0001)$, and did not result in any higher association with having an abnormal head CT either.

The prehospital GCS was obtained for all 946 patients transported via ambulance. It ranged from 3 to 15 , with a median of 15 and an interquartile range of 15-15. Thus, the majority of the patients had a prehospital GCS of 15 . However, $3 \%$ had a prehospital GCS of 13 , and $17 \%$ had a 14. A total of 838 patients were brought in by ambulance, while 86 were brought in by helicopter. More people brought in by helicopter $(48 \%)$ vs ambulance $(21 \%)$ had a prehospital GCS $<15(\mathrm{P}<0.0001)$. Regardless, having a prehospital GCS $<15$ was significantly associated with being admitted to the hospital $(P<$ $0.001)$ and having an abnormal CT scan $(\mathrm{P}<0.001)$.

A third of the cohort (30\%) were admitted to the hospital, of which 192 (29\%) were admitted to the Intensive Care Unit (ICU). Univariate predictors of hospital admission included LOC, AOC, and PTA, all $p$ $<0.0001$. In multivariate analysis, only LOC and PTA retained statistical significance. Having a lower prehospital GCS was also significantly associated with being admitted to the hospital $(p<0.0001,95 \% \mathrm{CI}$ -0.0677 to -0.1523 ). However, none of the symptoms or prehospital GCS were significantly associated with ICU admission.

Compared to the general population, patients admitted to the ICU (Table 2) were significantly older, with a median age of $62(\mathrm{IQR}=41-78)$. Patients admitted to the ICU were significantly more likely to be on an antiplatelet or anticoagulant agent $(p<0.0001,95 \% \mathrm{CI}-0.3153$ to - 0.4047) with warfarin, aspirin and clopidogrel retaining independent statistical significance. Patients admitted to the ICU were also significantly more likely to have experienced PTA ( $p=0.0025,95 \%$ CI -0.0352 to -0.1648$)$, LOC $(p<0.0001,95 \%$ CI -0.3282 to -0.4318$)$ or have an abnormal brain CT $(\mathrm{p}<0.0001,95 \%$ CI -0.6584 to 0.7816).

Patients who died in the hospital (Table 3) were significantly more likely to be on an antiplatelet or anticoagulant agent: warfarin $(P=0.0051,95 \% \mathrm{CI}-0.042$ to $-0.238)$, aspirin $(p=0.0005,95 \%$ CI -0.1139 to 0.4061 ), or clopidogrel ( $\mathrm{p}<0.0001,95 \%$ CI -0.1214 to 0.3186). All patients in the hospital death group had intracranial hemorrhage on ED head CT, despite having

Table 2 Factors associated with ICU admission

\begin{tabular}{|c|c|c|c|c|c|c|c|c|c|c|c|c|c|c|}
\hline & Age & Gender & $\begin{array}{l}\text { War- } \\
\text { farin }\end{array}$ & ASA & $\begin{array}{l}\text { Pla- } \\
\text { vix }\end{array}$ & $\begin{array}{l}\text { Any } \\
\text { AC }\end{array}$ & Fall & MVC & $\begin{array}{l}\text { PH GCS } \\
<15\end{array}$ & $\begin{array}{l}\text { Head } \\
\mathrm{CT}\end{array}$ & LOC & Vomit & PTA & $\mathrm{AOC}$ \\
\hline $\begin{array}{l}\text { Total cohort minus } \\
\text { ICU N=2019 }\end{array}$ & $\begin{array}{l}\text { Median 35, range 18- } \\
101 \text {, IQR 23-54 }\end{array}$ & $\begin{array}{l}57 \% \\
\text { male }\end{array}$ & $0,0 \%$ & $\begin{array}{l}106 \\
5 \%\end{array}$ & $\begin{array}{l}40, \\
2 \%\end{array}$ & $\begin{array}{l}146, \\
7 \%\end{array}$ & $\begin{array}{l}939 \\
47 \%\end{array}$ & $\begin{array}{l}689 \\
34 \%\end{array}$ & $\begin{array}{l}160 / 946 \\
17 \%\end{array}$ & $\begin{array}{l}315 \\
14 \%\end{array}$ & $\begin{array}{l}212 \\
11 \%\end{array}$ & $\begin{array}{l}115 \\
6 \%\end{array}$ & $\begin{array}{l}504 \\
25 \%\end{array}$ & $\begin{array}{l}153 \\
8 \%\end{array}$ \\
\hline $\begin{array}{l}\mathrm{ICU} \\
N=192\end{array}$ & $\begin{array}{l}\text { Median } 62 \text {, IQR 41-78, } \\
\text { range 18-95 }\end{array}$ & $\begin{array}{l}59 \% \\
\text { male }\end{array}$ & $\begin{array}{l}16 \\
8 \%\end{array}$ & $\begin{array}{l}42 \\
22 \%\end{array}$ & $\begin{array}{l}25 \\
13 \%\end{array}$ & $\begin{array}{l}83 \\
43 \%\end{array}$ & $\begin{array}{l}122 \\
64 \%\end{array}$ & $\begin{array}{l}57 \\
30 \%\end{array}$ & & $\begin{array}{l}170 \\
88 \%\end{array}$ & $\begin{array}{l}95 \\
49 \%\end{array}$ & $\begin{array}{l}13 \\
7 \%\end{array}$ & $\begin{array}{l}67 \\
35 \%\end{array}$ & $\begin{array}{l}65 \\
3 \%\end{array}$ \\
\hline
\end{tabular}


Table 3 Factors associated with in-hospital death

\begin{tabular}{|c|c|c|c|c|c|c|c|c|c|c|c|c|c|c|}
\hline & Age & Gender & Warfarin & ASA & Plavix & $\begin{array}{l}\text { Any } \\
\text { AC }\end{array}$ & Fall & MVC & $\begin{array}{l}\mathrm{PH} \\
\mathrm{GCS}< \\
15\end{array}$ & $\begin{array}{l}\text { Head } \\
\mathrm{CT}\end{array}$ & LOC & Vomit & PTA & $\overline{A O C}$ \\
\hline $\begin{array}{l}\text { Total cohort minus in } \\
\text { hosp death } N=2199\end{array}$ & $\begin{array}{l}\text { Median 37, IQR 23- } \\
56 \text {, range 18-101 }\end{array}$ & $\begin{array}{l}57 \% \\
\text { male }\end{array}$ & $\begin{array}{l}59 \\
3 \%\end{array}$ & $\begin{array}{l}144 \\
7 \%\end{array}$ & $\begin{array}{l}62 \\
3 \%\end{array}$ & $\begin{array}{l}146 \\
7 \%\end{array}$ & $\begin{array}{l}1052 \\
48 \%\end{array}$ & $\begin{array}{l}744 \\
34 \%\end{array}$ & $\begin{array}{l}189 \\
9 \%\end{array}$ & $\begin{array}{l}1984, \\
90 \%\end{array}$ & $102,447 \%$ & $\begin{array}{l}125 \\
6 \%\end{array}$ & $\begin{array}{l}568 \\
26 \%\end{array}$ & $\begin{array}{l}503 \\
23 \%\end{array}$ \\
\hline $\begin{array}{l}\text { Death } \\
N=12\end{array}$ & $\begin{array}{l}\text { Median } 81, \text { IQR } 70- \\
85, \text { range } 18-92\end{array}$ & $\begin{array}{l}50 \% \\
\text { male }\end{array}$ & $2,17 \%$ & $\begin{array}{l}4 \\
33 \%\end{array}$ & $\begin{array}{l}3 \\
25 \%\end{array}$ & $\begin{array}{l}9 \\
75 \%\end{array}$ & $\begin{array}{l}9 \\
75 \%\end{array}$ & $\begin{array}{l}2 \\
17 \%\end{array}$ & $\begin{array}{l}1 \\
8 \%\end{array}$ & $\begin{array}{l}12 \\
100 \%\end{array}$ & $\begin{array}{l}7 \\
58 \%\end{array}$ & $\begin{array}{l}3 \\
75 \%\end{array}$ & $\begin{array}{l}3 \\
25 \%\end{array}$ & $\begin{array}{l}1 \\
8 \%\end{array}$ \\
\hline
\end{tabular}

presented to the ED with a GCS of 15, and all but one patient also having a pre-hospital GCS of 15. Patients were also significantly more likely to have presented with vomiting ( $\mathrm{p}<0.0001,95 \% \mathrm{CI}-0.5514$ to -0.8286$)$ and were older with a median age of 81 , compared to the survivor cohort median age of 37 .

Patients who underwent neurosurgical intervention (Table 4$)$ were significantly more likely to be male $(P=$ $0.0203)$, to be on an antiplatelet or anticoagulant agent: warfarin $(\mathrm{P}=<0.0001)$, aspirin $(P<0.0001)$, or clopidogrel $(P=.0003)$. They were also more likely to have suffered their TBI from a fall $(P=0.0349)$, and experienced vomiting afterwards $(P=0.0193)$. Fourteen patients had their anticoagulant status reversed. 11 received IV vitamin $\mathrm{K}, 7$ received both IV vitamin $\mathrm{K}$ and fresh frozen plasma (FFP), 2 received FFP only, and 1 received SC vitamin $\mathrm{K}$.

A total of 1996 or $90 \%$ of patients had a brain CT scan. Of these, 485 or $24 \%$ had a CT abnormality (Table 5). The frequency of specific CT abnormalities is summarized in Fig. 3. The most common CT lesions noted on CT were extra-calvarial soft tissue swelling (41\%), parenchymal or hemorrhagic contusion (26\%), subdural hematoma (25\%), and subarachnoid hemorrhage (22\%). Patients who had an abnormal CT scan were significantly more likely to be older, on an anticoagulant, and have suffered a fall as their TBI mechanism (all $P<0.001)$.

\section{Discussion}

In this observational study of emergency department presentations for patients with a GCS of 15 upon arrival, a number of findings emerge that suggest that the simple characterization of head injury by GCS score may not be sufficient.

The cohort is somewhat unique in the proportion of patients who received a brain CT as part of their evaluation. Indeed, there are several rules that specifically seek to limit the use of head CT for head injury, within certain parameters. The Canadian head CT [18] rule excludes people on blood thinners, and those who had a seizure after the head injury. Also, a number of high-risk criteria have been noted with the rule, including use of blood thinners, any suspicion of skull fracture, age $>65$, and a change in GCS level. Medium risk criteria including a "dangerous mechanism" is also noted as a caveat. The New Orleans head CT criteria [19] actually targets only the GCS 15 head injury population, and "suggests patients with GCS 15 and head trauma are unlikely to need a head CT as long as they do not have headache, vomiting alcohol or drug intoxication, persistent anterograde amnesia, seizure, visible trauma above the clavicles."

Clearly, neither of these rules are optimal for the mild TBI cohort in this emergency department study. Studies published years after these rules concur, suggesting that "Patients with GCS 15 and risk factors or neurological symptoms should be evaluated with $\mathrm{CT}$ scan, [as] the outcome of mild TBI depends on the combination of preinjury, injury and postinjury factors" [20]. CT abnormalities are in fact not uncommon in mild TBI. A study of 2766 patients with mild TBI imaged in ED found that every sixth patient (16.1\%) with mild TBI had an intracranial lesion [21]. The most common lesions were

Table 4 Factors associated with neurosurgical intervention

\begin{tabular}{|c|c|c|c|c|c|c|c|c|c|c|c|c|c|c|}
\hline & Age & Gender & Warfarin & ASA & Plavix & $\begin{array}{l}\text { Any } \\
\text { AC }\end{array}$ & Fall & MVC & $\begin{array}{l}\text { ph GCS } \\
<15\end{array}$ & $\begin{array}{l}\text { Head } \\
\mathrm{CT}\end{array}$ & LOC & Vomit & PTA & $A O C$ \\
\hline $\begin{array}{l}\text { Total cohort } \\
\text { minus surgery } \\
N=2158\end{array}$ & $\begin{array}{l}\text { Median } 36, \text { IQR 23-56, } \\
\text { range 18-101 }\end{array}$ & $\begin{array}{l}56 \% \\
\text { male }\end{array}$ & $52,2 \%$ & $\begin{array}{l}137 \\
6 \%\end{array}$ & $\begin{array}{l}59 \\
3 \%\end{array}$ & $\begin{array}{l}220 \\
10 \%\end{array}$ & $\begin{array}{l}1028 \\
48 \%\end{array}$ & $\begin{array}{l}742 \\
34 \%\end{array}$ & $\begin{array}{l}200 / 917 \\
22 \%\end{array}$ & $\begin{array}{l}1944, \\
90 \%\end{array}$ & $\begin{array}{l}1016, \\
47 \%\end{array}$ & $\begin{array}{l}121 \\
6 \%\end{array}$ & $\begin{array}{l}561 \\
26 \%\end{array}$ & $\begin{array}{l}491 \\
23 \%\end{array}$ \\
\hline $\begin{array}{l}\text { Surgery } \\
N=53\end{array}$ & $\begin{array}{l}\text { Median } 64, \text { IQR } 41-78 \text {, } \\
\text { range } 20-86\end{array}$ & $\begin{array}{l}72 \% \\
\text { male }\end{array}$ & $9,17 \%$ & $\begin{array}{l}11 \\
21 \%\end{array}$ & $\begin{array}{l}6 \\
11 \%\end{array}$ & $\begin{array}{l}21 \\
40 \%\end{array}$ & $\begin{array}{l}33 \\
62 \%\end{array}$ & $4,8 \%$ & $\begin{array}{l}8 / 20 \\
40 \%\end{array}$ & $\begin{array}{l}52 \\
98 \%\end{array}$ & $\begin{array}{l}15 \\
28 \%\end{array}$ & $\begin{array}{l}7, \\
13 \%\end{array}$ & $\begin{array}{l}10 \\
19 \%\end{array}$ & $\begin{array}{l}13 \\
25 \%\end{array}$ \\
\hline
\end{tabular}


Table 5 Factors associated with an abnormal CT scan

\begin{tabular}{|c|c|c|c|c|c|c|c|c|c|c|c|c|c|}
\hline & Age & Gender & $\begin{array}{l}\text { Warfarin } \\
\text { med }=0\end{array}$ & $\begin{array}{l}\text { ASA } \\
\text { med }=1\end{array}$ & $\begin{array}{l}\text { Plavix } \\
\text { Med }=2\end{array}$ & $\begin{array}{l}\text { Any } \\
\text { AC }\end{array}$ & Fall & MVC & $\begin{array}{l}\text { ph GCS } \\
<15\end{array}$ & LOC & Vomit & PTA & AOC \\
\hline $\begin{array}{l}\mathrm{CT} \\
\text { normal } \\
n=1511\end{array}$ & $\begin{array}{l}\text { Median } 33 \text {, IQR 22-51, } \\
\text { range 18-95 }\end{array}$ & $\begin{array}{l}57 \% \\
\text { male }\end{array}$ & $30,2 \%$ & $52,3 \%$ & $17,1 \%$ & $\begin{array}{l}95 \\
6 \%\end{array}$ & $\begin{array}{l}678 \\
45 \%\end{array}$ & $\begin{array}{l}545 \\
36 \%\end{array}$ & $\begin{array}{l}127 / 639 \\
20 \%\end{array}$ & $\begin{array}{l}752 \\
50 \%\end{array}$ & $\begin{array}{l}88 \\
6 \%\end{array}$ & $\begin{array}{l}405 \\
27 \%\end{array}$ & $\begin{array}{l}297 / 1365 \\
22 \%\end{array}$ \\
\hline $\begin{array}{l}C T \\
\text { abnormal } \\
n=485\end{array}$ & $\begin{array}{l}\text { Median } 59 \text {, IQR 37-77, } \\
\text { range 18-101 }\end{array}$ & $\begin{array}{l}63 \% \\
\text { male }\end{array}$ & $31,6 \%$ & $85,18 \%$ & $45,9 \%$ & $\begin{array}{l}134 \\
28 \%\end{array}$ & $\begin{array}{l}307 \\
63 \%\end{array}$ & $\begin{array}{l}119 \\
25 \%\end{array}$ & $\begin{array}{l}84 / 248 \\
34 \%\end{array}$ & $\begin{array}{l}245 \\
51 \%\end{array}$ & $\begin{array}{l}34 \\
7 \%\end{array}$ & $\begin{array}{l}152 \\
31 \%\end{array}$ & $\begin{array}{l}151 / 481 \\
31 \%\end{array}$ \\
\hline
\end{tabular}

subdural hematomas, subarachnoid hemorrhages, and contusions. Similar to the current study, the authors noted that a lower Glasgow Coma Scale score, male sex, older age, falls, and chronic alcohol abuse were associated with higher risk of acute intracranial lesion in patients with mild TBI. These findings underscore the heterogeneity of neuropathology associated with the mild TBI classification.

Even in cases of mTBI, patients sometimes require neurosurgical intervention (NSI), as noted in the current study. In their study of mTBI patients, Tierney et al. [22] also noted that $8.2 \%$ had some form of NSI performed (including placement of an intracranial pressure monitor and other measuring devices). The in-hospital death rate for the NSI group was $13 \%$, significantly higher than general figures for mTBI ranging from $0.3-1.8 \%$. The use of anticoagulants as well as age being over 60 predicted a bad neurological outcome.
ICU admission for mTBI, while seemingly counterintuitive, is not all that uncommon. A retrospective study of 595,171 mTBI patients in the National Trauma Data Bank found that $44.7 \%$ were admitted to an ICU, While $17.3 \%$ of these met the criteria for overtriage, as defined by: ICU stay $\leq 1$ day; hospital stay $\leq 2$ days; no intubation; no neurosurgery; and discharged to home [23], that still leaves $27.4 \%$ of mTBI patients who were deemed appropriate for ICU admission. Interestingly, the study noted that a common "risk factor" for overtriage included isolated subarachnoid hemorrhage.

\section{Conclusion}

This study underscores: 1) the importance of considering neuroimaging in all patients with TBI, including those with a GCS 15. In the current study, a total of $10 \%$ of the cohort was not imaged. If the CT data is

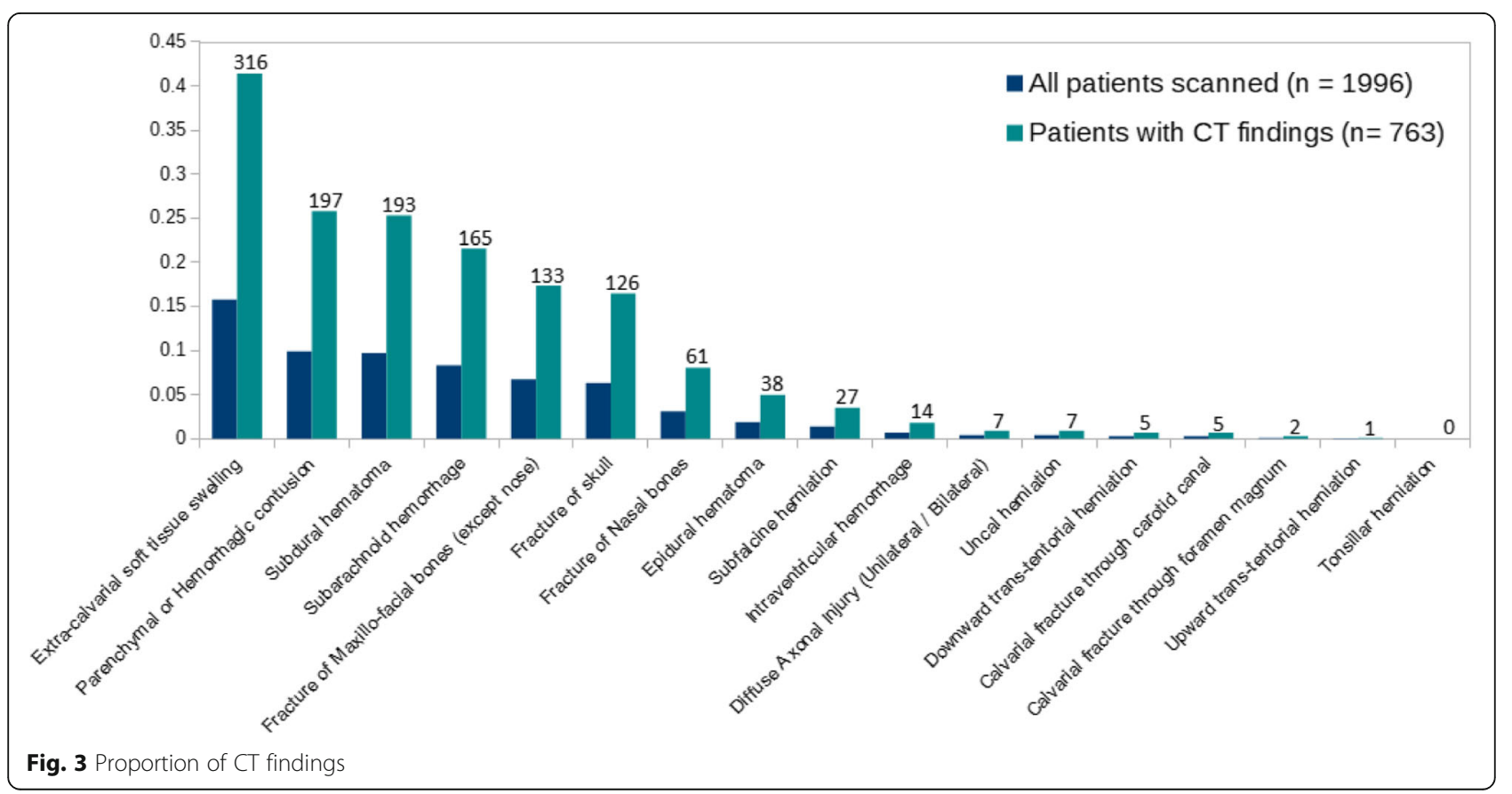




\section{Appendix}

Table 6 Study variables

\begin{tabular}{|c|c|c|c|}
\hline & Variable / Field Name & Field choices & $\begin{array}{l}\text { N complete (out of } \\
2211 \text { unless noted } \\
\text { otherwise) }\end{array}$ \\
\hline ed_arrivel_date & ED Arrival Date & date & 2211 \\
\hline age_in_years & Age & number in years & 2211 \\
\hline sex & Gender & 0, Female $\mid 1$, Male & 2211 \\
\hline race & Race & 0, Caucasian | 1, Black | 2, Hispanic | 3, Native American | 4, Asian | 5, Other & 2211 \\
\hline marital_status & Marital Status & 0, Single | 1, Married | 2, Divorced/ Separated | 3, Not known & 2210 \\
\hline employment & Employment Status & 0, Not employed | 1, Employed | 2, Retired | 3, Student | 4, Not known & 2656 \\
\hline date_head_injury & Date of Head Injury & date & 1959 \\
\hline time_head_injury & Time of Head Injury & $0,0-12 \mathrm{~h}$ ago $\mid 1,12-24 \mathrm{~h}$ ago $\mid 2$, More than $24 \mathrm{~h}$ ago & 2192 \\
\hline head_injury_reporting & Head Injury Reporting & 0 , Self Reported | 1, Friend/ family | 2, EMS & 2211 \\
\hline asso_vomit & Vomiting after injury & 0 , No $\mid 1$, Yes & 2210 \\
\hline asso_seizure & Seizures after injury & 0, No $\mid 1$, Yes & 2209 \\
\hline loss_consciousness & Loss of Consciousness & 0, No $\mid 1$, Yes $\mid 2$, Unknown & 2211 \\
\hline aoc & $\begin{array}{l}\text { Altered Mental Status/Alteration } \\
\text { of Consciousness (AOC) }\end{array}$ & 0, No $\mid 1$, Yes $\mid 2$, Unknown & 2211 \\
\hline pta & Post Traumatic Amnesia (PTA) & 0, No $\mid 1$, Yes $\mid 2$, Unknown & 2211 \\
\hline fall & Association of Fall with Injury & 0, No $\mid 1$, Yes $\mid 2$, Unknown & 2211 \\
\hline occupational & Was this an occupational injury? & 0, No $\mid 1$, Yes $\mid 2$, Unknown & 2210 \\
\hline hit_struck_fall_head & $\begin{array}{l}\text { Did anything hit/struck/fall on } \\
\text { head? }\end{array}$ & 0, No $\mid 1$, Yes $\mid 2$, Unknown & 2211 \\
\hline sport & $\begin{array}{l}\text { Did the injury occur while } \\
\text { playing a sport? }\end{array}$ & 0, No $\mid 1$, Yes $\mid 2$, Unknown & 2211 \\
\hline recreational_activity & $\begin{array}{l}\text { Injury occurred during a } \\
\text { recreational activity? }\end{array}$ & 0, No $\mid 1$, Yes $\mid 2$, Unknown & 2210 \\
\hline rec_vehicle & $\begin{array}{l}\text { Was a recreational vehicle } \\
\text { involved? }\end{array}$ & 0, No $\mid 1$, Yes $\mid 2$, Unknown & $379 / 379$ \\
\hline rec_vehicle_type & $\begin{array}{l}\text { What type of Rec Vehicle was } \\
\text { involved? }\end{array}$ & 0, Bicycle $\mid$ 1, Motorcycle $\mid 2$, ATV | 3, Other $\mid$ 4, Watercraft | 5, Not Known & $290 / 290$ \\
\hline helmet_use & $\begin{array}{l}\text { Use of Helmet in Recreational } \\
\text { Vehicle injury }\end{array}$ & 0, No $\mid 1$, Yes $\mid 2$, N/A & $284 / 290$ \\
\hline traffic_accident & $\begin{array}{l}\text { Did the Injury occur in a traffic } \\
\text { accident? }\end{array}$ & 0, No $\mid 1$, Yes $\mid 2$, Unknown & 2211 \\
\hline traffic_accident_driver & Location of Patient & $\begin{array}{l}0 \text {, Driver } \mid \text { 1, Front Seat Passenger | 2, Back Seat Passenger | 3, Open Air Seating | } \\
\text { 4, Other } \mid 5 \text {, Unknown }\end{array}$ & 746 \\
\hline seatbelt & Was a seatbelt worn? & $0, \mathrm{No}|1, \mathrm{Yes}| 2, \mathrm{~N} / \mathrm{A}$ & 742 \\
\hline alcohol_bf_injury & $\begin{array}{l}\text { Alcohol Consumption Prior to } \\
\text { Injury (by Patient) }\end{array}$ & 0, No $\mid 1$, Yes $\mid 2$, Unknown & 2211 \\
\hline assaulted & Was the patient Assaulted? & 0, No $\mid 1$, Yes $\mid 2$, Unknown & 2211 \\
\hline location_injury_occured & $\begin{array}{l}\text { At what location did the injury } \\
\text { occur? }\end{array}$ & $\begin{array}{l}\text { 0, Inside a Home } \mid \text { 1, Outside a home (yard/driveway) } \mid \text { 2, On the road | } 3 \text {, } \\
\text { "Outdoors" } \mid \text { 4, At Work } \mid 5 \text {, Restaurant or Bar/Club } \mid 6 \text {, Store | 7, Other Public Area } \\
\text { (Park/sidewalk) | 8, Other }\end{array}$ & 2211 \\
\hline transfer & $\begin{array}{l}\text { Was the patient transferred from } \\
\text { another facility? }\end{array}$ & 0, No $\mid 1$, Yes & 2211 \\
\hline mode_trans & Mode of Transportation & 0, Walk in/private vehicle $\mid 1$, Ambulance | 2, Helicopter & 2211 \\
\hline admitted_dis_or & Discharged or Admitted & 0, Discharged | 1, Admitted & 2211 \\
\hline hlos & Hospital Length of Stay & number value & $666 / 666$ \\
\hline icu & ICU Stay? & 0, No $\mid 1$, Yes & $666 / 666$ \\
\hline icu_los & ICU Length of Stay & number value & 192/192 \\
\hline disch_disposition & Discharge Disposition & $\begin{array}{l}\text { 1, routine } d c \mid 2 \text {, rehab } \mid 3 \text {, psych } \mid \text { 4, expired } 5 \text {, hospice/NH| 6, AMA } 7 \text {, law } \\
\text { enforcement }\end{array}$ & 2211 \\
\hline in_hospital_death & In hospital death? & 0, No $\mid 1$, Yes & 2211 \\
\hline prehosp_gcs_completion & Pre Hospital Glasgow Coma & 0, No $\mid 1$, Yes & $946 / 1381$ \\
\hline
\end{tabular}


Table 6 Study variables (Continued)

\begin{tabular}{|c|c|c|c|}
\hline & Variable / Field Name & Field choices & $\begin{array}{l}\mathrm{N} \text { complete (out of } \\
2211 \text { unless noted } \\
\text { otherwise) }\end{array}$ \\
\hline & Scale Completion & & \\
\hline & Pre Hospital GCS & number from 3 to 15 & \\
\hline tbi_severity_prehosp & TBI Severity- Pre Hospital & 0 , Mild $\mid 1$, Moderate $\mid 2$, Severe & $946 / 1381$ \\
\hline ed_gcs_completion & $\begin{array}{l}\text { ED Glasgow Coma Scale } \\
\text { Completion }\end{array}$ & 0, No $\mid 1$, Yes & 2211 \\
\hline ed_gcs_score_1 & ED GCS Score & number from 3 to 15 & 2211 \\
\hline tbi_severity_ed & TBI Severity- ED & 0 , Mild $\mid 1$, Moderate $\mid 2$, Severe & 2211 \\
\hline surgical_intervention & Surgical intervention done? & 0 , No $\mid 1$, Yes & 2211 \\
\hline ct_scan & CT Scan Done? & 0, No $\mid 1$, Yes & 2211 \\
\hline head_ct_abmormal & Head CT abnormal? & 0 , No 1 , Yes & 1996/1996 \\
\hline ct_findings & $\begin{array}{l}\text { Please check any/all } \\
\text { abnormalities that are observed } \\
\text { on the CT scan: }\end{array}$ & $\begin{array}{l}\text { 0, Extra-calvarial soft tissue swelling | 1, Fracture of skull | 2, Fracture of Maxillo- } \\
\text { facial bones (except nose) | 3, Fracture of Nasal bones | 4, Calvarial fracture } \\
\text { through carotid canal | 5, Calvarial fracture through foramen magnum | 6, Subfal- } \\
\text { cine herniation } \mid 7 \text {, Upward trans-tentorial herniation | 8, Downward trans- } \\
\text { tentorial herniation } \mid 9 \text {, Uncal herniation | 10, Tonsillar herniation | 11, Epidural } \\
\text { hematoma } \mid 12 \text {, Subdural hematoma | 13, Subarachnoid hemorrhage | 14, Intra- } \\
\text { ventricular hemorrhage | 15, Parenchymal or Hemorrhagic contusion | 16, Diffuse } \\
\text { Axonal Injury (Unilateral / Bilateral) }\end{array}$ & $485 / 485$ \\
\hline alcohol_level & Alcohol Blood Level & number value & 233 \\
\hline urine_drug_screen & Urine Drug Screen & 0 , Negative $\mid 1$, Positive & 157 \\
\hline arr_meds & $\begin{array}{l}\text { Arrival Anticoagulant } \\
\text { Medications? }\end{array}$ & 0 , No 1 , Yes & 2167 \\
\hline med_type & Medications- Types & $\begin{array}{l}\text { 0, Warafrin | 1, Aspirin | 2, Plavix | 3, Aggrenox | 4, Ticlid | 5, Dipyridamole | 6, } \\
\text { Heparin | 7, Enoxaparin | 8, Dabigatran }\end{array}$ & $2167 / 2167$ \\
\hline anticoag_rev_int & $\begin{array}{l}\text { Anticoagulation Reversal } \\
\text { Intervention? }\end{array}$ & 0 , No $\mid 1$, Yes & $189 / 242$ \\
\hline int_type & Intervention- Type & $\begin{array}{l}\text { 0, IV Vitamin K | 1, FFP | 2, PCC | 3, Factor IX Complex | 4, Recombinant Factor VIla } \\
\mid \text { 5, SC Vitamin K } \mid \text { 6, Other }\end{array}$ & $14 / 14$ \\
\hline
\end{tabular}

extrapolated to the non-imaged group, the proportion of bleeds and fractures would be increased by 2.5 and 1.5 percentage points, respectively. 2) recognizing the limitations of GCS in classifying TBI, as patients with even the mildest of mild TBI have a high frequency of gross CT abnormalities, and non-benign outcomes including ICU admission, neurosurgical interventions and even in-hospital death.

\section{Abbreviations}

AOC: Alteration of consciousness; CT: Computer tomography; ED: Emergency department; GCS: Glasgow coma score; ICU: Intensive care unit; LOC: Loss of consciousness; PTA: Post-traumatic amnesia; RedCap: Research Electronic Data Capture; TBI: Traumatic brain injury

\section{Acknowledgements}

None.

\section{Funding}

n/a

\section{Availability of data and materials}

$\mathrm{n} / \mathrm{a}$ (retrospective chart review).

\section{Authors' contributions}

$L G, A N B$ and $Y D$ conceived the study. $L G, Y D, A N B, S A$, and $C P$ collected the data. $L G$, and ANB performed the statistical analyses and cross checked the data. TS and LG drafted the manuscript, and all authors contributed substantially to its revision. KP supervised the conduct of the research and data collection. LG takes responsibility for the paper as a whole. All authors read and approved the final manuscript.

\section{Ethics approval and consent to participate}

This retrospective chart review study was approved by the Institutional

Review Board at the University of Florida, study\#188-2011.

\section{Consent for publication}

Waiver of informed consent was approved by the IRB for this retrospective chart review.

\section{Competing interests}

None of the authors have any completing interests.

\section{Publisher's Note}

Springer Nature remains neutral with regard to jurisdictional claims in published maps and institutional affiliations.

\section{Author details}

${ }^{1}$ UCF HCA Emergency Medicine Residency Program of Greater Orlando, University of Central Florida College of Medicine, Orlando, FL, USA. ${ }^{2}$ Polk County Fire Rescue, University of Central Florida, Orlando, FL, USA. ${ }^{3}$ University of Central Florida, Orlando, USA. ${ }^{4}$ New York College of Podiatric Medicine, New York, NY, USA. ${ }^{5}$ Department of Neurology, Saint Louis University, Saint Louis, MO, USA. ${ }^{6}$ Department of Emergency Medicine, University of Cincinnati, Cincinnati, OH, USA. ${ }^{7}$ Touro College of Osteopathic Medicine, Mare Island, CA, USA. ${ }^{8}$ Division of Neuroradiology, University of Florida, Gainesville, FL, USA. 
Received: 15 October 2018 Accepted: 26 December 2018

Published online: 28 February 2019

\section{References}

1. Faul M, Xu L, Wald MM, et al. Traumatic brain in the United States: Emergency department visits, hospitalizations and deaths, 2002-2006. Atlanta, GA: Centers for Disease Control and Prevention, National Center for Injury Control and Prevention; 2010.

2. Centers for disease control, prevention. Report to congress on mild traumatic brain injury in the United States: steps to prevent a serious health problem. Atlanta, GA: Centers for Disease Control and Prevention, National Center for Injury Prevention and Control; 2003.

3. DoD Worldwide Numbers for TBI. https://www.brainline.org/article/howmany-servicemembers-have-sustained-tbi\#annualdiagnoses. Accessed 25 Jan 2019.

4. Dean, P. J., \& Sterr, A. (2013). Long-term effects of mild traumatic brain injury on cognitive performance. Frontiers in Human Neuroscience, 7, 30.T

5. Ganti, L., Daneshvar, Y., Ayala, S., Bodhit, A. N., \& Peters, K. R. (2016). The value of neurocognitive testing for acute outcomes after mild traumatic brain injury. Military Medical Research, 3, 23. https://doi.org/10.1186/s40779016-0091-4.

6. Middelboe, T., Anderson, H. S., Birket-Smith, M., \& Friis, M. L. (1992). Mino head injury: Impact on general health after 1 year. A prospective follow-up study. Acta Neurologica Scandinavica, 5, 5-9.

7. Ganti, L., Daneshvar, Y., Bodhit, A., Ayala, S., Patel, P. S., Lottenberg, L. L., York, D., Counsell, C., \& Peters, K. R. (2015). TBI ADAPTER: Traumatic brain injury assessment diagnosis advocacy prevention and treatment from the emergency room-a prospective observational study. Military Medicine, 180(4), 380-386. https://doi.org/10.7205/MILMED-D-14-00316.

8. De Kruijk, J. R., Leffers, P., Menheere, P. P., Meerhoff, S., Rutten, J., \& Twijnstra, A. (2002). Prediction of post-traumatic complaints after mild traumatic brain injury: Early symptoms and biochemical markers. Journal of Neurology, Neurosurgery, and Psychiatry, 73(6), 727-732.

9. Ganti, L., Khalid, H., Patel, P. S., Daneshvar, Y., Bohdit, A. N., \& Peters, K. R. (2014). Who gets post-concussion syndrome? An emergency departmentbased prospective analysis. International Journal of Emergency Medicine, 7, 31. https://doi.org/10.1186/s12245-014-0031-6

10. Auxéméry, Y. (2012). Mild traumatic brain injury and postconcussive syndrome: A re-emergent questioning. Encéphale, 38(4), 329-335.

11. Ganti, L., Conroy, L., Bodhit, A., et al. (2015). Understanding why patients return to the emergency department after mild traumatic brain injury within 72 hours. WestJem. https://doi.org/10.5811/westjem.2015.2.23546.

12. Teasdale, G., \& Jennett, B. (1974). Assessment if coma and impaired consciousness. A practical scale. Lancet, 2, 81-84.

13. Teasdale, G., \& Jennett, B. (1976). Assessment and prognosis of coma after head injury. Acta Neurochirgica, 34, 45-55.

14. Saatman, K. E., Duhaime, A.-C., Bullock, R., Maas, A. I. R., Valadka, A., \& Manley, G. T. (2008). Classification of traumatic brain injury for targeted therapies. Journal of Neurotrauma, 25(7), 719-738. https://doi.org/10.1089/neu.2008. 0586.

15. Kay, T., Harrington, D. E., Adams, R., et al. (1993). Definition of mild traumatic brain injury. The Journal of Head Trauma Rehabilitation, 8(3), 86-87.

16. Si, B., Dumkrieger, G., Wu, T., Zafonte, R., Valadka, A. B., Okonkwo, D. O., et al. (2018). Sub-classifying patients with mild traumatic brain injury: A clustering approach based on baseline clinical characteristics and 90-day and 180-day outcomes. PLoS One, 13(7), e0198741 https://doi.org/10.1371/journal.pone. 019874116.

17. Stead, L. G., Bodhit, A. N., Patel, P. S., et al. (2013). TBI surveillance using the common data elements for traumatic brain injury: A population study. International Journal of Emergency Medicine., 6, 5. https://doi.org/10.1186/ 1865-1380-6-5.

18. Stiell, I. G., et al. The Canadian CT Head Rule for patients with minor head injury. The Lancet, 357(9266), 1391-1396.

19. Haydel, M. J., Preston, C. A., Mills, T. J., Luber, S., Blaudeau, E., \& DeBlieux, P. M. C. (2000). Indications for computed tomography in patients with minor head injury. The New England Journal of Medicine, 343, 100-105.

20. Shukla, D., \& Devi, B. I. (2010). Mild traumatic brain injuries in adults. Journal of Neurosciences in Rural Practice., 1(2), 82-88. https://doi.org/10.4103/09763147.71723

21. Isokuortti, H., Iverson, G. L., Silverberg, N. D., Kataja, A., Brander, A., Öhman, J. \& Luoto, T. M. (2018 Jan). Characterizing the type and location of intracranial abnormalities in mild traumatic brain injury. Journal of Neurosurgery, 12, 1-10. https://doi.org/10.3171/2017.7.JNS17615.

22. Tierney, K. J., Nayak, N. V., Prestigiacomo, C. J., \& Sifri, Z. C. (2016). Neurosurgical intervention in patients with mild traumatic brain injury and its effect on neurological outcomes. Journal of Neurosurgery, 124(2), 538-545. https://doi.org/10.3171/2015.4.JNS142440 Epub 2015 Sep 25.

23. Bonow, R. H., \& Quistberg, A. (2018). Rivara. Neurocrit Care: F.P. et al https://doi.org/10.1007/s12028-018-0590-0.
Ready to submit your research? Choose BMC and benefit from:

- fast, convenient online submission

- thorough peer review by experienced researchers in your field

- rapid publication on acceptance

- support for research data, including large and complex data types

- gold Open Access which fosters wider collaboration and increased citations

- maximum visibility for your research: over $100 \mathrm{M}$ website views per year

At $\mathrm{BMC}$, research is always in progress.

Learn more biomedcentral.com/submissions 